\title{
Comparison of tests for the detection of circulating filarial antigen (Og4C3-ELISA and AD12-ICT) and ultrasound in diagnosis of lymphatic filariasis in individuals with microfilariae
}

\author{
Abraham Rocha ${ }^{1}{ }^{+}$, Cynthia Braga ${ }^{1}$, Marcela Belém¹, Arturo Carrera' ${ }^{1}$ Ana Aguiar-Santos ${ }^{1}$, \\ Paula Oliveira', Maria José Texeira', André Furtado² \\ ${ }^{2}$ Departamento de Entomologia ${ }^{1}$ Departamento de Parasitologia, Serviço de Referência Nacional em Filarioses e Serviço de Referência \\ em Culicídeos Vetores, Centro de Pesquisas Aggeu Magalhães-Fiocruz, Av. Moraes Rego s/n, 50670-420 Recife, PE, Brasil
}

Significant advances were made in the diagnosis of filariasis in the 1990s with the emergence of three new alternative tools: ultrasound and tests to detect circulating antigen using two monoclonal antibodies, Og4C3 and AD12-ICT-card. This study aimed to identify which of these methods is the most sensitive for diagnosis of infection. A total of 256 individuals, all male and carrying microfilariae $(1-15,679 \mathrm{MF} / \mathrm{mL})$, diagnosed by nocturnal venous blood samples, were tested by all three techniques. The tests for circulating filarial antigen concurred 100\% and correctly identified 246/256 (96.69\%) of the positive individuals, while ultrasound detected only 186/256 (73.44\%). Of the circulating antigen tests, ICT-card was the most convenient method for identification of Wuchereria bancrofti carriers. It was easy to perform, practical and quick.

Key words: Wuchereria bancrofti - circulating filarial antigen - ultrasound - diagnosis

Lymphatic filariasis (LF) is a parasitic disease caused by nematodes (Wuchereria bancrofti, Brugia malayi and Brugia timori) whose preferred habitats are the lymphatic vessels and lymph nodes which induce the development of disfiguring and debilitating clinical symptoms. The infection, which is transmitted by various genera of mosquitoes (Sasa 1976), is considered to be one of the health problems of greatest social and economic impact in endemic areas (Ottesen et al. 1997).

Until the 1980s, the only direct way to confirm a diagnosis of infection by $W$. bancrofti was via the identification of microfilariae (MF) in peripheral blood using camera counting, polycarbonate membrane filtration, the thick smear method or Knott's technique (Knott 1939, Denham et al. 1971, Dennis \& Kaen 1971, Eberhard \& Lammie 1991). Apart from the low sensitivities of these methods arising from the fact that they only identify filarial infection by way of microfilaremia, they are also inconvenient in terms of the time of day the blood must be collected, namely when the MF are at a peak in peripheral blood, a parameter that varies from one endemic area to another. In Brazil, the peak for microfilaremia occurs between 11 pm-1 am (Dreyer et al. 1996).

In the 1990s, significant advances were made in the diagnosis of LF with the emergence of new diagnostic

Financial support: FIOCRUZ/FUNASA (projects 268-369-433, subproject 17), FIOTEC/SVS (projects 573-17 and 801-17), Departamento de Parasitologia do Centro de Pesquisas Aggeu Magalhães

+ Corresponding author: rocha@cpqam.fiocruz.br

Received 2 December 2008

Accepted 22 June 2009 tools: (i) use of recombinant antigens to detect specific antibodies (Chandrasherkar et al. 1994); (ii) a PCR for the detection of filarial DNA (Zhong et al. 1996, Rocha et al. 2002); (iii) the visualisation of live adult worms (AW) using ultrasound (US) and (iv) circulating filarial antigen (CFA) detection (More \& Copeman 1990, Weil et al. 1997). At present, the standard diagnostic tools are US (Amaral et al. 1994) and CFA detection, with the latter using the monoclonal antibodies (McAbs) $\mathrm{Og} 4 \mathrm{C} 3$ and AD12. The CFA detection techniques are commercially available in the form of kits and have the advantage of allowing for diagnosis to be carried out using blood samples collected at any time of day (More \& Copeman 1990, Amaral et al. 1994, Weil et al. 1997, Rocha 2002, 2004).

$\mathrm{Og} 4 \mathrm{C} 3$, the first commercially available laboratory kit, is a serological test based on the detection of CFA using an immunoenzymatic technique (ELISA) (More \& Copeman 1990) and a McAb of the IgM class of immunoglobulins produced against antigens of the bovine parasite Onchocerca gibsoni. This technique allows for the identification of $W$. bancrofti antigens in serum, plasma and hydrocele fluid and does not show any cross-reactivity with other helminthic infections (More \& Copeman 1990, Rocha et al. 1996, TropBIO 1996, Rocha 2004).

The other serological test, the immunochromatographic or ICT card test, uses the McAb AD12, which recognises a $200 \mathrm{kDa}$ filarial antigen (Weil et al. 1997). The technique is easy to perform, requires no equipment and was launched by ICT Diagnostic (Balgowlah, New South Wales, Australia). It is currently produced by BINAX as NOW ${ }^{\circledR}$ Filariasis in the United States of America.

Another tool for diagnosis of filariasis infection, first discovered by Brazilian researchers, is the use of US to visualise live AW of $W$. bancrofti in the lymphatic vessels (Amaral et al. 1994, Norões et al. 1996). 
Although several authors attest to the high sensitivity of these new methods for diagnosing filarial infection by $W$. bancrofti, a comparative analysis of the sensitivity of these techniques has not been performed.

\section{PATIENTS, MATERIALS AND METHODS}

Area and population studied - This study was carried out on 256 male patients with microfilaremia who were residents of endemic areas in the Metropolitan Region of Recife and referred to the National Center of Lymphatic Filariasis (NCLF)'s outpatient clinic at the Aggeu Magalhães-Research Centre Oswaldo Cruz Foundation (Fiocruz). The patients selected for this study were seen between 2001-2004.

Parasitological blood test - After participants had signed the terms of consent, a sample of $5 \mathrm{~mL}$ of venous blood was collected between $11 \mathrm{pm}-1$ am in order to test for the presence of circulating MF. A quantity of $1 \mathrm{~mL}$ of blood was filtered through a polycarbonate membrane $13 \mathrm{~mL}$ in diameter with $3-\mu \mathrm{m}$ pores, as described by Rocha et al. (2004).

Test for live $A W$ - All of the 256 individuals underwent an US of the scrotal area using a $7.5 \mathrm{MHz}$ probe to visualise the live AW and identify their characteristic movements in lymphatic vessels, i.e., the filariae dance sign (FDS) (Amaral et al. 1994, Norões et al. 1996).

Collection of serum samples - One day after venous blood was collected for detection of the presence of MF, an additional sample of $5 \mathrm{~mL}$ of venous blood was collected and centrifuged at $2,500 \mathrm{rpm}$ at $20^{\circ} \mathrm{C}$; the serum was separated, quantified, labelled and stored at $-20^{\circ} \mathrm{C}$ in the NCLF's biological specimen bank. In order to detect filarial antigens, the serum from each individual was thawed in an ice bath and the technical procedures for the CFA tests were carried out and processed using a single technique. In comparison, a single serum sample from each individual was exposed to both CFA tests.

The Og4C3-ELISA test - The technical procedures were carried out as recommended by the manufacturers of the kit (TropBio ${ }^{\circledR}$, JCU Tropical Biotechnology Pty Ltd, Townsville, Queensland, Australia). Serological samples were processed in duplicate and the results were obtained using optical density (OD) measurement. The OD measurements for each sample were used to determine the response in units of antigen per $\mathrm{mL}(\mathrm{ag} / \mathrm{mL})$ by comparison to a standard concentration curve for $O$. gibsoni $\mathrm{ag} / \mathrm{mL}$, included in the kit. In accordance with the TropBio norms (1996) samples with $\mathrm{ag} / \mathrm{mL} \geq 128$ were considered positive for CFA.

The AD12-ICT card test - The NOW ${ }^{\circledR}$ Filariasis version of the card test (ICT filariasis for blood, serum or plasma, cat. $\mathrm{n}^{\circ}$. 620-000) was carried out according to the manufacturer's instructions. In short, $100 \mu \mathrm{L}$ of serum was placed in the position recommended for the test. After one minute, the card was closed and the result was read exactly 10 min later. The test was considered positive when both lines (test and control) could be read through the visualisation window. Any line (light or dark) appearing in the test position indicates that the result of the test is positive; it is negative when the control line can be seen.

Statistical analysis - The difference between the proportions was verified using the chi-squared test and the $p$ value at a level of $\alpha=0.05$. Owing to the large variation in values for the units of circulating antigen per $\mathrm{mL}$, the differences between the means were tested using the Kruskall-Wallis test.

Ethical considerations - This study was approved by the Aggeu Magalhães-Fiocruz Research Centre's Ethical Committee. The participants (or their parents in the case of minors) were given information about the research and were asked to read and sign the terms of consent. All participants were treated with diethylcarbamazine (6 mg/kg/day/12 days).

\section{RESULTS}

The age of individuals varied from 7-78 years (mean $=24.14 \pm 10.13)$. The density of MF per $\mathrm{mL}$ of blood was between 1-15,679 (mean $=768.56 \pm 1.520 .24)$ and the antigenemia levels by $\mathrm{Og} 4 \mathrm{C} 3$ varied between $1-56.411 \mathrm{ag} /$ $\mathrm{mL}($ mean $=28.328 .87 \pm 14.932 .78)$.

The percentages for positive results for the three tests are shown in Table I. The sensitivities of the two techniques for detection of CFA (Og4C3 and ICT card) were similar, both showing a high degree of sensitivity $(96.69 \%)$. The percentage of US positive results was $73.44 \%\left(\chi^{2}=4.21 ; p=0.046\right)$. Live AW was not detected in $68(26.56 \%)$ individuals through the US exam.

The relation between the density of $\mathrm{MF} / \mathrm{mL}$ and the quantitative and qualitative results of the $\mathrm{Og} 4 \mathrm{C} 3$ tests (unit of ag/mL) and the ICT card (positive/negative), respectively, showed a significant increase in the antigen level in Og4C3 CFA and in the percentage of positive tests using the ICT card proportional to the rise in the parasite load chiefly when the microfilaremia density was greater than $100 \mathrm{MF} / \mathrm{mL}$ of blood (Tables II, III).

The comparison between US and the density of MF per $\mathrm{mL}$ showed that the percentage of tests in which the FDS could be seen significantly increased in proportion to the number of MF. In the patients with a microfilarial density of $>500 \mathrm{MF} / \mathrm{mL}$, AW could be visualised by US in $82.56 \%$ (Table IV).

\section{TABLE I}

Percentage of positive results and confidence interval (CI) for the ICT card, $\mathrm{Og} 4 \mathrm{C} 3$ and ultrasound tests among $\mathrm{MF}^{a}{ }^{a}$ mean

\begin{tabular}{lccc}
\hline $\begin{array}{l}\text { Test } \\
(\mathrm{n}=256)\end{array}$ & Positives & $\%$ & CI 95\% \\
\hline Og4C3-ELISA & 246 & 96.69 & $93.15-97.99$ \\
ICT card test & 246 & 96.69 & $93.16-97.99$ \\
Ultrasound & 188 & 73.44 & $67.77-78.57$ \\
\hline
\end{tabular}

$a$ : individuals with microfilaremia diagnosed using the polycarbonate membrane filtration technique; $\chi^{2}=4.21 ; p=0.046$. 
A higher proportion of positive results by US was observed in individuals between 20-30 (132/157) and $>30$ years $(21 / 35)$ of age compared to the group between 7-19 years $(35 / 64)$ of age $\left(\chi^{2}=23.88, p<0.0001\right)$. In the CFA test results $(\mathrm{Og} 4 \mathrm{C} 3$ and ICT card), no statistically significant difference in the proportion of positive results among these age groups was observed $\left(\chi^{2}=0.63, p<0.731\right)$.

\section{TABLE II}

Average of $\mathrm{Og} 4 \mathrm{C} 3$ antigenemia levels according to density of microfilariae

\begin{tabular}{lccccc}
\hline Density & \multicolumn{5}{c}{ Og4C3 antigenemia $(\mathrm{ag} / \mathrm{mL})$} \\
\hline Microfilariae/mL & $\mathrm{n}$ & Mean $^{a}$ & Min & Max & $\begin{array}{c}\text { Standard } \\
\text { deviation }\end{array}$ \\
\hline $1-99$ & 78 & 20,517 & 1 & 50,485 & 15,136 \\
$100-500$ & 82 & 29,089 & 3 & 53,333 & 14,707 \\
$>500$ & 86 & 35,497 & 1,415 & 56,411 & 10,546 \\
Total & 246 & 28,328 & 1 & 56,411 & 14,933 \\
\hline
\end{tabular}

$a$ : arithmetic mean. Kruskal Wallis $(2 \mathrm{gL})=52.00 ; \mathrm{p}=0.0001$.

\section{TABLE III}

Percentage of positive results using the ICT card according to the parasite load of microfilariae among individuals carrying microfilariae

\begin{tabular}{lcccc}
\hline Density & \multicolumn{4}{c}{ ICT card } \\
\hline Microfilariae/mL & Positives & $\%$ & Negatives & Total \\
\hline $1-99$ & 78 & 89.66 & 9 & 87 \\
$100-500$ & 82 & 98.80 & 1 & 83 \\
$>500$ & 86 & 100.0 & 0 & 87 \\
Total & 246 & 96.67 & 10 & 256 \\
\hline
\end{tabular}

$\chi^{2}$ test for trend $=12.32 ; \mathrm{p}=0.0004$.

\section{TABLE IV}

Frequency of filariae dance sign detected by ultrasound of the scrotum according to microfilariae parasite load in individuals carrying microfilariae

\begin{tabular}{lcccc}
\hline Density & \multicolumn{4}{c}{ Ultrasound (live adult worm) } \\
\hline Microfilariae/mL & Positive & $\%$ & Negative & Total \\
\hline $1-99$ & 55 & 63.22 & 32 & 87 \\
$100-500$ & 62 & 74.70 & 21 & 83 \\
$>500$ & 71 & 82.56 & 15 & 86 \\
Total & 188 & 73.44 & 68 & 256 \\
\hline
\end{tabular}

$\chi^{2}$ test for trend $=8.266 ; \mathrm{p}=0.0040$.

\section{DISCUSSION}

Recent advances in various areas of knowledge related LF have led to the use of new approaches to lessen the severity and impact of the disease (Ottesen et al. 1997). The introduction of new diagnostic tools for detection of infection by $W$. bancrofti in the human population have opened up new possibilities in terms of interrupting transmission and consequently the elimination of filariasis (WHO 1994, Cox 2000, Molyneux et al. 2000, Ottesen 2000). This was reaffirmed in 1997 by the World Health Organisation (WHO), when it established the goal of filariasis elimination worldwide by 2020 (Ottesen 2000).

Until the early 1990 s, the only available parasitological method for the evaluation of individuals or populations infected with $W$. bancrofti was the blood thick smear, which uses around $60 \mu \mathrm{L}$ of capillary blood (Eberhard \& Lammie 1991). However, due to its low sensitivity, this method does not allow accurate determination of the true prevalence of infection (McCarthy 2000). With the introduction of the filtration technique and the use of venous blood (up to $10 \mathrm{~mL}$ ) collected between $11 \mathrm{pm}$ $1 \mathrm{am}$, a higher degree of reliability of the parasitological status of individuals was obtained (Rocha 2004).

The introduction of US in Recife, Brazil, as a new parasitological tool for diagnosis of bancroftosis made it possible to identify individuals with live AW of $W$. bancrofti in the lymphatic vessels of the scrotal sac (Amaral et al. 1994). Several studies have emphasised the importance of US as a non-invasive parasitological tool (Dreyer et al. 1995, 1998, Faris et al. 1998). The data presented here showed that the use of US was capable of identifying AW in $73.44 \%$ of MF+ individuals. This result is similar to that of Norões et al. 1996, who identified AW in $80 \%$ of MF+ individuals using US.

The CFA method was more sensitive than the US method in identifying infected individuals. This result is possibly due to the fact that the AW could be located in sites of the body other than male genitals, such as the scrotum, which has been described as the preferred location of AW (Amaral et al. 1994).

Due to the simplicity and convenience of this method, it is possible to carry out the test using blood collected at any time of day. CFA is now considered to be a promising tool for diagnosis of bancroftosis at both the individual and community levels. Among CFA tests, the ICT card, because of its simplicity and precision, has been adopted as an important tool for community surveys and rapid assessment of filarial endemicity in bancroftian filariasis control programs (Ottesen 2000, Molyneux \& Taylor 2001). Nevertheless, in large surveys, it can be difficult to examine the cards while at the same time handling many patients, resulting in a tendency of health care providers to gather the cards to be read at a later time. This procedure could contribute to misinterpretation of the results and could increase the number of false positives. In our study, the ICT card test failed to detect CFA in nine of 78 individuals with MF count less than $100 \mathrm{MF} / \mathrm{mL}$, while US failed to detect live AW in 32 of 87 individuals carrying MF. There is concern that currently available tools may have severe limitations when parasite prevalence and antigen levels are low. 
Og4C3 and AD12 McAbs detect circulating $W$. bancrofti antigens originating particularly from AW, according to More and Copeman (1990) and Weil et al. (1997). Furthermore, Eberhard et al. (1997) and Lalitha et al. (1997) suggest that the MF do not contribute to the concentration of CFA and that the decrease in the quantity of CFA occurs exclusively as a result of the death of AW. Wong and Guest (1969) noted that microfilarial antibodies were present in all patients with elephantiasis, but were found in none of the patients with circulating MF. Smithers (1968) has suggested that living MF are relatively inert antigenically and do not stimulate the production of antibodies. Our results contradict these observations and clearly show that there is a significant association $(p=0.001)$ between the density of MF and the concentration of CFA. Similar results were obtained by Rocha et al. (1996), Nicolas et al. (1997) and Itoh et al. (1999). We also agree with Grove and Davis (1978), who suggest that the detection of microfilarial antibodies depends upon the relative release of MF into the bloodstream and the production of antibodies against those MF.

Medeiros et al. (1999) showed that men between 20-30 years of age are more susceptible to LF than are women of the same age group. This was also the largest age group in our study $(\sim 60 \%)$.

The sensitivity of US was lower (73.44\%) than that of the monoclonal $\mathrm{Og} 4 \mathrm{C} 3(96.69 \%)$ and $\mathrm{AD} 12$ (96.69\%) techniques. On the other hand, for age groups 20-30 and $>30$ years, the US was statistically significantly ( $p>$ 0.0001) effective in detecting AW. Therefore, in order to provide a rapid and reliable detection of infection with $W$. bancrofti, the choice of diagnostic tool must take into consideration age-group, gender, the presence of infected individuals harbouring low levels of MF, the presence of growing worms or non-fecund adults or adults of a single sex and the endemicity of the area.

In summary, in our study, McAbs $\mathrm{Og} 4 \mathrm{C} 3$ and $\mathrm{AD} 12$ showed high sensitivity for detecting infected individuals. While the global LF elimination program is ongoing, highly sensitive and specific diagnostic tools are necessary to monitor and control the programs. There is presently no "gold standard technique" that alone is capable of offering complete confidence that infection has been eliminated and that can by its use alone allow the WHO to certify an area free of LF. The Og4C3 ELISA and the ICT card test, in our study, presented high performance in detecting positive individuals. Nevertheless, both have advantages and inconveniences with respect to their use in large control programs. The ICT card test may be most appropriate, because of its ease of use and because results are obtained within $10 \mathrm{~min}$. In cases where one needs to broaden the diagnostic investigation, US should be of great value, as demonstrated here.

Until now, no CFA test available has been reported to be $100 \%$ specific in detecting infection by $W$. bancrofti (Rocha et al. 1996).

\section{ACKNOWLEDGEMENTS}

To Dr. Patrick Lammie, for the critical review of the manuscript and helpful comments.

\section{REFERENCES}

Amaral F, Dreyer G, Figueredo JS, Norões J, Cavalcanti A, Samico SC, Santos A, Coutinho A 1994. Adult worms detected by ultrasonography in human bancroftian filariasis. Am J Trop Med Hyg 50: $753-757$.

Chandrashekar R, Curtis KC, Ramzy RM, Liftis FLBW, Weil GJ, 1994. Evaluation of a recombinant antigen-based antibody assay for diagnosis of bancroftian filariasis in Egypt. Mol Biochem Parasitol 64: 261-271.

Cox FEG 2000. Elimination of lymphatic filariasis as a public health problem. Parasitol Today 16: 135-137.

Denham DA, Dennis DT, Ponnudurai T, Nelson GS, Guy F 1971. Compararison of a counting chamber and thick blood smear methods of counting microfilariae. Trans $\mathrm{R}$ Soc Trop Med Hyg 65: 521-526.

Dennis DT, Kaen BH 1971. Isolation of microfilariae: report of new method. J Parasitol 57: 1146-1147.

Dreyer G, Norões J, Amaral F, Nen A, Medeiros Z, Coutinho A, Addiss D 1995. Direct assessment of the adulticidal efficacy of a single dose of invermectin in bancroftian filariasis. Trans $R$ Soc Trop Med Hyg 2: 441-443.

Dreyer G, Pimentel A, Medeiros Z, Béliz F, Galdino E, Moura E, Coutinho A, Andrade LD, Rocha A, Silva LM. Piessens WF 1996. Studies on the periodicity and intravasular distribution of Wuchereria bancrofti microfilariae in paired samples of capillary and venous blood from Recife. Brazil. Trop Med Int Health 1: 264-272.

Dreyer G, Santos A, Norões J, Amaral F, Addiss D 1998. Ultrasonographic detection of living adult Wuchereria bancrofti using a 3,5 MHz transducer. Am J Trop Med Hyg 9: 399-403.

Eberhard ML, Hightower AW, Addiss DG, Lammie PJ 1997. Clearence of Wuchereria bancrofti antigen after treatment winth diethylcarbamazine or ivermectin. Am J Trop Med Hyg 57: 483-486.

Eberhard ML, Lammie PJ 1991. Laboratory diagnosis of filariasis. Clin Lab Med 11: 977-1010.

Faris R, Hussain O, El-Setouhy M, Ramzy RM, Weil GB 1998. Bancroftian filariasis in Egypt: visualization of adult worms and subclinical lymphatic pathology by scrotal ultrasound. Am J Trop Med Hyg 59: 864-867.

Grove DI, Davis RS 1978. Serological diagnosis of bancroftian and Malayan filariasis. Am J Trop Med Hyg 27: 508-513.

Itoh M, Weerasooriya MV, Gunawardena NK, Mudalige MPS, Samarawickrema WA, Kimura E 1999. Wuchereria bancrofti antigenemia in Sri Lanka. Trop Med Int Health 4: 207-210.

Knott JA 1939. Method for making microfilarial surveys on day blood. Trans R Soc Trop Med Hyg 33: 191-196.

Lalitha P, Ravichandran M, Suba S, Kaliraj P, Narayanan RB, Jayaraman K 1997. Quantitative assessment of circulating antigens in human lymphatic filariasis: a field evaluation of monoclonal antibody-based ELISA using blood collected on filter strips. Trop Med Int Health 3: 41-45.

McCarthy J 2000. Diagnosis of lymphatic filarial infections. In: TB Nutman, Lymphatic filariasis, Imperial College Press, London, p. 127-150.

Medeiros Z, Gomes J, Béliz F, Coutinho A, Dreyer P, Dreyer G 1999. Screening of army soldiers for Wuchereria bancrofti infection in the metropolitan Recife region, Brazil: implications for epidemiological surveillance. Trop Med Int Health 4: 499-505.

Molyneux DH, Neira M, Liese B, Heymann 2000. Elimination of lymphatic filariasis as a public health problem. Trans $R$ Soc Trop Med Hyg 94: 589-591. 
Molyneux DH, Taylor MJ 2001. Current status and future prospects of the Global Lymphataic Filariasis Programme. Curr Opin Infect Dis 14: 155-159.

More SJ, Copeman DB 1990. A highly specific and sensitive monoclonal antibody-based ELISA for the detection of circulating antigen in bancroftian filariasis. Trop Med Parasitol 41: 403-406.

Nicolas L, Plichart C, Nguyen LN, Moulia-Pelat J 1997. Reduction of Wuchereria bancrofti adult worm circulating antigen after annual treatments of diethylcarbamazine combined with ivermectin in French Polynesia. J Infect Dis 175: 489-492.

Norões J, Addis D, Amaral F, Coutinho A, Medeiros Z, Dreyer G 1996. Occurrence of living adult Wuchereria bancrofti in scrotal area of men with microfilaremia. Trans $R$ Soc Trop Med Hyg 90: $55-56$.

Ottesen EA 2000. The global programme to eliminate lymphatic filariasis. Trop Med Int Health 5: 591-594.

Ottesen EA, Duke BOL, Karam M, Behbehani K 1997. Strategies and tools for the control/elimination of lymphatic filariasis. Bull World Health Organ 75: 491-503.

Rocha A 2000. Métodos laboratoriais disponíveis para o diagnóstico da filariose linfática. Rev Soc Bras Anal Clin 32: 265-270.

Rocha A 2004. Filariose bancroftiana: avaliação dos testes de diagnósticos disponiveis frente às diversas formas clínicas da bancroftose, PhD Thesis, Instituto Oswaldo Cruz-Fiocruz, Rio de Janeiro, $234 \mathrm{pp}$.

Rocha A, Addis D, Ribeiro ME, Norões J, Baliza M, Medeiros Z, Dreyer G 1996. Evaluation of the Og4C3 ELISA in Wuchereria bancrofti infection: infected persons with undetectable or ultralow microfilarial densities. Trop Med Int Health 1: 859-864.
Rocha A, Junqueria AC, Furtado A 2002. Molecular approach in the diagnosis of lymphatic filariasis by Wuchereria bancrofti. Rev Patol Trop 31: 161-174.

Rocha A, Lima G, Medeiros Z, Santos A, Alves S, Montarroyos U, Oliveira P, Béliz F, Netto M, Furtado A 2004. Circulating filarial antigen (CFA) in the hydrocele fluid from individuals living in a bancroftian filariasis area-Recife-Brasil, detected by the monoclonal antibody Og4C3-assay. Mem Inst Oswaldo Cruz 99: 101-105.

Sasa M 1976. The antifilariasis campaign: its history and future prospects. In M Sasa, Human filariasis. A global survey of epidemiology and control, University Park Press of Tokyo, Tokyo, p. 3-11.

Smithers SR 1968. Immunity to blood helminths, 6th Symposium of the British Society for Parasitology, in AER, Taylor, Diagnosis of the causative organisms of tropical eosinophilia and filariasis, Blackweel, ed. Blackwell, Oxford, pages 55-66.

TropBIO 1996. ELISA kit for detecting and quantifying Wuchereria bancrofti antigen, Townsville, Australia: JCU Tropical Biotecnology Pty, James Cook University of North Queensland.

Weil GJ, Lammie PJ, Weiss N 1997. The ICT filariasis test: a rapidformat antigen test for diagnosis of bancroftian filariasis. Parasitol Today 13: 401-404.

WHO/CTD/TDR 1994. Strategies for control of lymphatic filariasis infection and disease: Report of a WHO/CTD/TDR consultative meeting held at the University Sains, Malaysia, Penang, Malaysia, p. 22-24.

Wong MM, Guest MF 1969. Filarial antibodies and eosinophilia in human subjects in an endemic area. Trans R Soc Trop Med Hyg 63: 796-800.

Zhong M Mccarthy J, Bierwert L, Lizotte-Waniewski M, Chanteau S, Nutman TB, Ottensen EA, Williams SA 1996. A polymerase chain reaction assay for detection of the parasite Wuchereria bancrofti in human blood samples. Am J Trop Med Hyg 54: 357-363. 